\title{
A Conference on the Origin (and Evolution) of Baryonic Galaxy Halos
}

\author{
Duncan Forbes ${ }^{1, *}$ and Ericson Lopez ${ }^{2}$ \\ 1 Centre for Astrophysics and Supercomputing, Swinburne University, Hawthorn VIC 3122, Australia \\ 2 Observatorio Astronomico de Quito, Escuela Politecnica Nacional, Quito 17-01-165, Ecuador; \\ ericsson.lopez@epn.edu.ec \\ * Correspondence: dforbes@swin.edu.au
}

Academic Editor: Emilio Elizalde

Received: 10 May 2017; Accepted: 10 May 2017; Published: 17 May 2017

\begin{abstract}
A conference was held in March 2017 in the Galapagos Islands on the topic of The Origin (and Evolution) of Baryonic Galaxy Halos. It attracted some 120 researchers from around the world. They presented 68 talks (nine of which were invited) and 30 posters over five days. A novel element of the talk schedule was that participants were asked which talks they wanted to hear and the schedule was made up based on their votes and those of the Scientific Organizing Committee SOC . The final talk schedule had $34 \%$ of the talks given by women. An emphasis was given to discussion time directly after each talk. Combined with limited/no access to the internet, this resulted in high level of engagement and lively discussions. A prize was given to the poster voted the best by participants. A free afternoon included organized excursions to see the local scenery and wildlife of the Galapagos (e.g., the giant tortoises). Four public talks were given, in Spanish, for the local residents of the town. A post-conference survey was conducted, with most participants agreeing that the conference met their scientific needs and helped to initiate new research directions. Although it was challenging to organize such a large international meeting in such an isolated location as the Galapagos Islands (and much credit goes to the Local Organizing Committee LOC and staff of Quito Astronomical Observatory for their logistical efforts, organizing the meeting for over a year), it was very much a successful conference. We hope it will play a small part in further developing astronomy in Ecuador.
\end{abstract}

Keywords: galaxies; formation; evolution; halos

\section{Conference Introduction}

Galaxy halos provide important clues to the origin and evolution of galaxies. We decided that the time was ripe to bring together the latest simulations and deep observations of galaxy halos in a conference focusing on the baryonic (star and gas) component of halos. In particular, deep, wide, and detailed observations of galaxy halos beyond the Local Group are becoming more ubiquitous. Simulations that incorporate realistic baryonic physics in a cosmological context have also made significant progress in recent years in modelling galaxy halos. These simulations predict outer halo regions that differ strongly in their formation processes and properties from the well-studied inner regions of galaxies. Halos have long dynamical times, and as such preserve the unique signatures of galaxy assembly.

Where better to focus on the Origin (and Evolution) of Baryonic Galaxy Halos than the Galapagos Islands? The islands were of course the inspiration for Charles Darwin's discovery on the origin and evolution of species. The islands are relatively cheap compared to some USA and European locations, and have the necessary infrastructure to guarantee a successful meeting. The key to a successful conference is the people and a conference structure that facilities presentations and discussion. In order to encourage the attendance of Ph.D. students, we completely waived the registration fee for students. 
We were very pleased to attract 120 researchers from all over the globe (i.e., Africa, Asia, Europe, North America, South America (including a dozen from Ecuador), and Australia). A photograph of the conference participants is shown in Figure 1. The conference included an official dinner and wildlife excursions for participants and their guests.

Another key element of choosing the Galapagos was to help promote Ecuadorian astronomy and the wider region. The conference received considerable media coverage, including television in Quito. We hope that the meeting provides a long-lasting legacy for astronomy in Ecuador. In an effort to bring astronomy to the Islands, two nights of public talks on astronomy were given in Spanish by Ricardo Salinas, Ericson Lopez, Wladimir Banda, and Carlos Frenk.

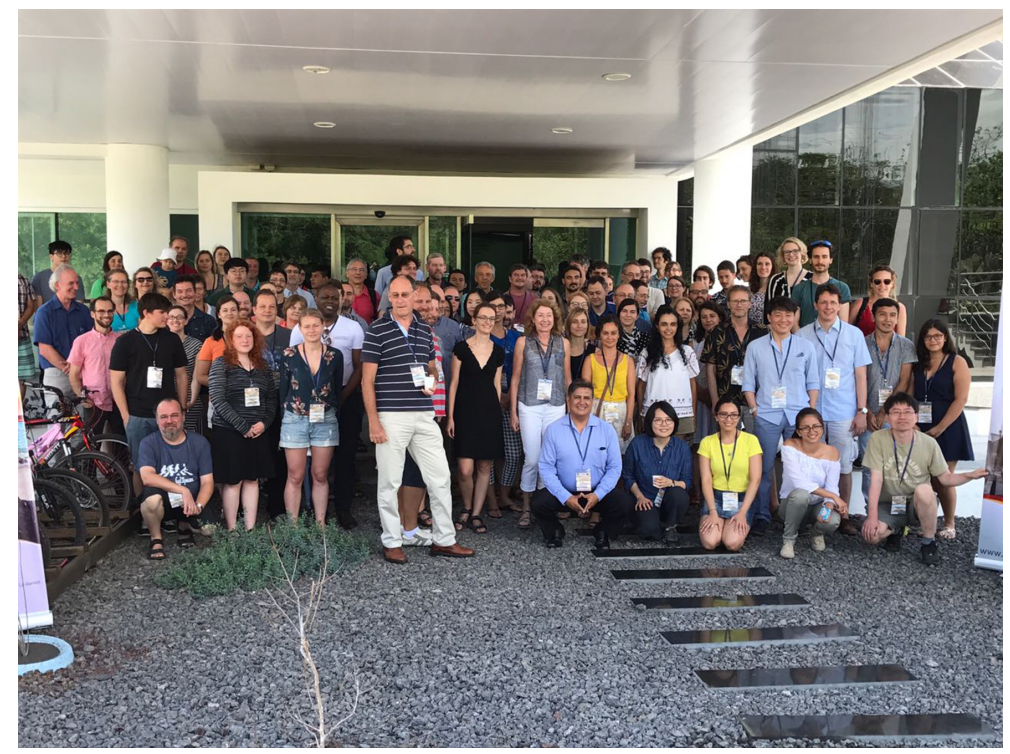

Figure 1. Conference photograph showing participants outside of the conference venue, the CIER (Centro de Informacion de Energia Renovable) building.

\section{Voting for Talks}

At the suggestion of SOC member Michael Merrifield, we tried an experiment to get participants more involved. Using Survey Monkey, we asked everyone registered to vote for their top 50 talks (including their own!). Some 2/3 of participants voted. The most voted for talk (the "people's choice") was by Evan Skillman. Combined with the votes from the SOC members, a draft schedule was made up. This schedule required only minor tweaking to ensure a balanced program. The final schedule consisted of 68 talks, with 23 by women (34\%, which also reflects the overall attendance fraction). Several talk slots were allocated to Ph.D. students. Overall, voting for the talk schedule received positive feedback from participants.

\section{Conference Structure}

The conference was held at the new Centro de Informacion de Energia Renovable (CIER)-the renewable energy center, part of the electricity company of Galapagos (Elecgalapagos)—in Puerto Ayora on the island of Santa Cruz in the Galapagos. A reception was held on the Sunday night, with the conference starting at 9 a.m. on Monday 13 March 2017. The conference was opened by Dr. Alberto Celi, the Vice-Rector for research and innovation at the Escuela Politecnica Nacional (EPN) University in Quito (host of the conference through its Quito Astronomical Observatory). A short welcome speech was also given by a representative from the local Ministry of Tourism. The basic format was invited talks of $25 \mathrm{~min}$ plus $10 \mathrm{~min}$ discussion, long talks $20 \mathrm{~min}$ plus $5 \mathrm{~min}$, and short talks of $10 \mathrm{~min}$ plus $5 \mathrm{~min}$. Thus, a key emphasis was placed on having plenty of time for discussion immediately after each talk. Indeed a notable aspect of the conference was the lively discussion throughout the meeting, which extended 
to the coffee breaks. Internet was generally unavailable during the conference (satellite service is very expensive on the islands), but many participants felt this contributed to a more engaged audience and lively discussion. Some 30 posters were also presented. A couple of coffee breaks were dedicated to poster time and a chance to chat with the author. Voting forms were given out to everyone, and the best poster was determined. A small prize was given to Gwendolyn Eadie at the end of the conference for her poster. Thus, dedicated coffee breaks and poster voting ensured engagement with the posters.

\section{Post-Conference}

Many participants chose to stay on after the conference and see the sights in the islands and/or in Quito. We followed up the conference by requesting PDF files of each talk so that participants and those unable to attend the conference can see the talks in detail at the conference homepage. In the longer-term, a conference proceedings book will be published. We also conducted a short survey to gauge the success of the conference with participants. When asked about the statement "The Galapagos galaxy halos conference meet my scientific needs", over 90\% said they Agreed or Strongly Agreed. Similarly, to the statement "The galaxy halos conference helped me to initiate new research project or direction", over 90\% Agreed or Strongly Agreed.

The articles that follow in this Special Issue include some of the talks and posters given at the conference. We hope that this forms a useful collection of works that reflects the current state-of-the-art on the Origin and Evolution of Baryonic Halos and promote the development of astronomical research in Ecuador and its neighboring countries.

Acknowledgments: We thank Escuela Politecnica Nacional, Centro de Educacion Continua (CEC-EPN), Parque Nacional Galapagos, and Centro de Informacion de Energia Renovable for their support. We would like to thank the Scientific Organising Committee: Jean Brodie, Carlos Frenk, Lars Hernquist, Claudia Mendes de Oliveira, Michael Merrifield, Marina Rejkuba and Aaron Romanowsky. We also thank the Local Organising Committee: Jairo Armijos, Wladimir Banda and Crispin Logan. Finally, a special thanks to the staff from Quito Astronomical Observatory for their logistical support that made the conference a great success.

Conflicts of Interest: The authors declare no conflict of interest.

(c) 2017 by the authors. Licensee MDPI, Basel, Switzerland. This article is an open access article distributed under the terms and conditions of the Creative Commons Attribution (CC BY) license (http:/ / creativecommons.org/licenses/by/4.0/). 